\title{
Dermatomiositis asociada al cáncer de mama: caracteristicas clínicas, complicaciones y tratamiento
}

\author{
Cristian David Sosa-Vesga* \\ Laura Marcela Pardo-Parra** \\ Sergio Eduardo Martínez-León** \\ David René Camargo Tarazona** \\ Álvaro Enrique Niño Rodríguez***
}

\begin{abstract}
* Médico interno. Estudiante XI nivel de medicina. Miembro del Grupo de Investigación de Cirugía y Especialidades (GRICES UIS). Universidad Industrial de Santander. Escuela de medicina. Facultad de salud. Bucaramanga. Santander. Colombia.

**Estudiante de VI nivel de medicina. Universidad Industrial de Santander. Escuela de medicina. Facultad de salud. Bucaramanga. Santander. Colombia. ***Médico cirujano especialista en cirugía de seno y tejidos blandos. Hospital Universitario de Santander. Docente del departamento de cirugía de la Universidad Industrial de Santander. Miembro del Grupo de Investigación de Cirugía y Especialidades (GRICES UIS). Escuela de medicina. Facultad de salud. Bucaramanga. Santander. Colombia.

Correspondencia: Cristian David Sosa Vesga. Dirección: carrera 32 \#29-31. Edificio de administración docente piso 5, oficina de Escuela de Medicina. Facultad de salud, Universidad Industrial de Santander. Bucaramanga. Santander. Colombia. Teléfono: (+57) 3013445873. Correo electrónico: csosa722@gmail.com.
\end{abstract}

Resumen

El cáncer de mama es un importante problema de salud pública. Aunque es infrecuente, uno de los síndromes paraneoplásicos del cáncer de seno es la dermatomiositis. Es necesario que el clínico tenga conocimientos sobre cómo diagnosticarla y tratarla adecuadamente. El objetivo de este artículo es realizar una revisión de la literatura sobre la clínica, complicaciones y tratamiento de la dermatomiositis en el cáncer de mama. Se realizó una búsqueda en diferentes bases de datos electrónicas, incluyendo un total de 34 artículos, abarcando ensayos clínicos aleatorizados, metaanálisis, artículos originales descriptivos y analíticos y reportes de caso. La dermatomiositis puede presentarse antes, durante, o después del cáncer de mama. Son frecuentes las complicaciones pulmonares, que pueden llegar a ser letales. La dermatomiositis aumenta las complicaciones por radioterapia y la mortalidad en los pacientes con cáncer de seno. El tratamiento farmacológico consiste en la aplicación de glucocorticoides a dosis según necesidad del paciente. MÉD.UIS. 2018;31(2):41-7.

Palabras claves: Dermatomiositis. Neoplasias de la Mama. Síndromes Paraneoplásicos.

\section{Dermatomyositis associated with breast cancer: Clinical characteristics, complications and treatment}

Abstract

Breast cancer is an important public health problem. Dermatomyositis is an infrequent paraneoplastic syndrome of breast cancer. It is necessary that the clinician has knowledge on how to diagnose and treat it properly. The aim of this article is to review the literature on the clinical, complications and treatment of dermatomyositis in breast cancer. A search was made in electronic databases, including a total of 34 articles, covering randomized clinical trials, meta-analyzes, original descriptive and analytical articles and case reports. Dermatomyositis can occur before, during, or after breast cancer. Pulmonary complications are frequent, and they can be lethal. Dermatomyositis increases the complications of radiotherapy and mortality in patients with breast cancer. The pharmacological treatment consists of glucocorticoid application at doses according to the patient's need. MÉD.UIS. 2018;31(2):41-7.

Keywords: Dermatomyositis. Breast Neoplasms. Paraneoplastic Syndromes.

¿Cómo citar este artículo?: Sosa-Vesga CD, Pardo-Parra LM, Martínez-León SE, Camargo DR, Niño AE. Dermatomiositis asociada al cáncer de mama: características clínicas, complicaciones y tratamiento. MÉD.UIS. 2018;31(2):41-7. Páginas. DOI: 10.18273/revmed.v31n2-2018005

Artículo recibido el 12 de julio del 2018 y aceptado para publicación el 14 de julio del 2018. 


\section{Introducción}

El cáncer de mama es un importante problema de salud pública. Según la Organización Mundial de la Salud, es el cáncer más frecuente en el género femenino, con un porcentaje aproximado del 16\%1. En el 2012, más de 408.000 mujeres fueron diagnosticadas con cáncer de seno en las américas y aproximadamente 92.000 de ellas fallecieron a causa de este ${ }^{2}$.

Según proyecciones de la Organización Panamericana de la Salud, el número de diagnósticos aumentará en un $46 \%$ para el año 2030. La proporción de defunciones por este tipo de cáncer en mujeres menores de 65 años es mayor en América Latina y el Caribe con el $57 \%$ que en Norteamérica con el $41 \%$. Aunque en los últimos años se han presentado avances científicos en su tratamiento, en muchos países de América Latina no se ha visto mejoría de su pronóstico, debido a que en varias regiones el acceso a estos avances aún es limitado ${ }^{2,3,4}$.

Según datos del Instituto Nacional de Cancerología de Colombia, entre el 2007 y el 2011, se presentó en el país una incidencia estimada anual ajustada por edad de cáncer de mama de 33,8 por cada 100 ooo habitantes, y una mortalidad anual ajustada por edad de 9,9 por cada 100000 habitantes 3,5 . En Bucaramanga, desde el 2008 al 2012 se presentaron cerca de 1295 casos de cáncer de mama, con una incidencia anual de 46,3 por cada 100000 habitantes $^{6}$.

La dermatomiositis (DM) es una entidad que se puede presentar de manera idiopática o como un síndrome paraneoplásico en un gran número de patologías tumorales, caracterizada por la presencia de un proceso inflamatorio muscular con manifestaciones cutáneas específicas ${ }^{7}$. Su incidencia no es clara y su presentación es poco frecuente de manera aislada, y un poco mayor cuando se presenta asociada a un proceso tumoral. Los tumores que más se asocian a la aparición de DM son los de ovario, colorrectales, seno y pulmón. Los mecanismos implicados en la relación entre la DM y el cáncer aún no son claros, pero se cree que este último induce una activación del complemento y la acción de la inmunidad humoral sobre los capilares musculares, lo cual tiene un papel crucial en la aparición de la enfermedad ${ }^{8,9,10}$.

Diversas alteraciones genéticas se relacionan con la DM. Estudios han reportado que anomalías en el locus del complejo mayor de histocompatibilidad (MHC), y alteraciones en el gen del antígeno leucocitario humano (HLA) DPB1 tienen un papel importante en la respuesta inmune de esta enfermedad, este último principalmente asociado a la presentación de antígeno de las moléculas HLA de clase II. Sin embargo, no se ha reportado en la literatura una asociación genética entre la DM y el cáncer de mama $^{11,12}$.

Aunque esta entidad es poco frecuente, es necesario que se conozca su correcto enfoque y manejo con el fin de evitar la aparición de complicaciones que pueden poner en riesgo la vida del paciente. El objetivo de este artículo es realizar una revisión de la literatura sobre las características clínicas y semiológicas de la DM enfocadas a los pacientes con cáncer de mama, describir las complicaciones que más frecuentemente se asocian con esta enfermedad y definir el tratamiento adecuado, con el fin de brindar una herramienta científica de conocimiento a todos los miembros del equipo de manejo interdisciplinario.

\section{Metodología de búsqueda}

Se realizó una búsqueda bibliográfica en PUBMED, EMBASE, OVID, MEDLINE, SCIELO y Google Scholar, usando los términos "dermatomyositis AND breast cancer", "treatment AND dermatomyositis" y "complications AND dermatomyositis". Los criterios de inclusión fueron: estudios con diseño metodológico de ensayos clínicos aleatorizados, metaanálisis, artículos originales descriptivos y analíticos y reportes de caso, que su población objetivo fuera mayor de 18 años, y cuyo tema principal se relacionara con la dermatomiositis. Se revisaron también las estadísticas de la Organización Mundial de la Salud, de la Agencia Internacional para la Investigación en Cáncer y reportes epidemiológicos del Instituto Nacional de Cancerología y el Ministerio de Salud y Protección Social de Colombia.

Se obtuvieron en total 147 resultados de los cuales se excluyeron 113 artículos por: no estar disponible su versión completa, estar publicados en un idioma diferente al español o al inglés y porque no aportaban información relevante para el objetivo de este artículo. Se obtuvieron al final 34 artículos, los cuales fueron revisados por los autores y se procedió a la redacción de este artículo. 


\section{Desarrollo del tema}

\section{Características clínicas}

Los síndromes paraneoplásicos pueden presentarse en aproximadamente 7 al $15 \%$ de los pacientes oncológicos y pueden anteceder las manifestaciones propias del tumor por meses, presentarse concomitantemente con la neoplasia, o aparecer tiempo después de la resolución del mismo ${ }^{13,14}$. Es por esto que, al presentarse un caso de DM la sospecha clínica y evaluación de una posible patología tumoral maligna deberán ser aspectos importantes por considerar en el abordaje del paciente ${ }^{14}$.

Estos síndromes aparecen frecuentemente como antesala al descubrimiento de una neoplasia y en la mayoría de los casos su sintomatología continúa la trayectoria evolutiva del tumor y se controla cuando éste lo hace. Tumores de pulmón, linfomas, cáncer de mama, ovario, tumores gastrointestinales y renales, son en los que más frecuentemente se presenta la DM como síndrome paraneoplásico ${ }^{13}$.

La DM es una inflamación miopática proximal perteneciente a un grupo de enfermedades autoinmunes y sistémicas, caracterizándose principalmente por la inflamación del músculo estriado y manifestaciones cutáneas. Estas manifestaciones son: rash heliotropo, edema periorbitario, pápulas violáceas sobre los nudillos, telangiectasias, cutículas distorsionadas con dilataciones capilares, calcificaciones cutáneas y fotosensibilidad, entre otros (Ver Figura 1 y Figura 2) $13,15,16$.

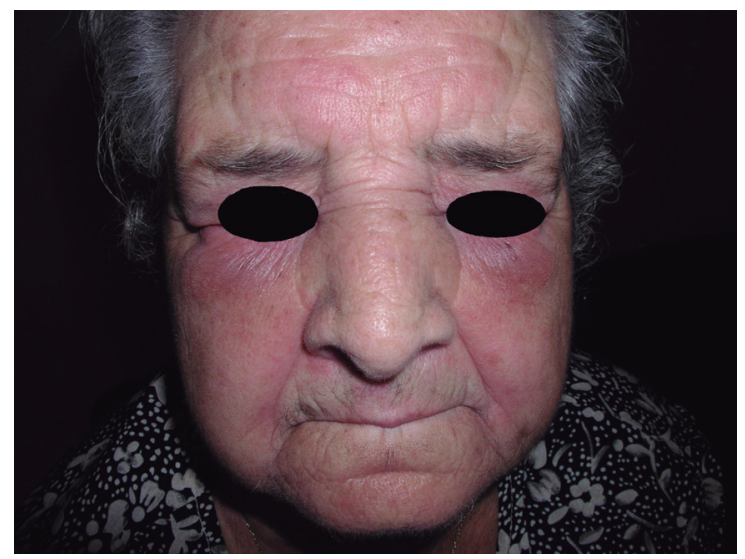

Figura 1: Rash Heliotropo

Fuente: Ciudad-Blanco C. et al. Actas Dermosifiliogr. 2011;102(6)448.55

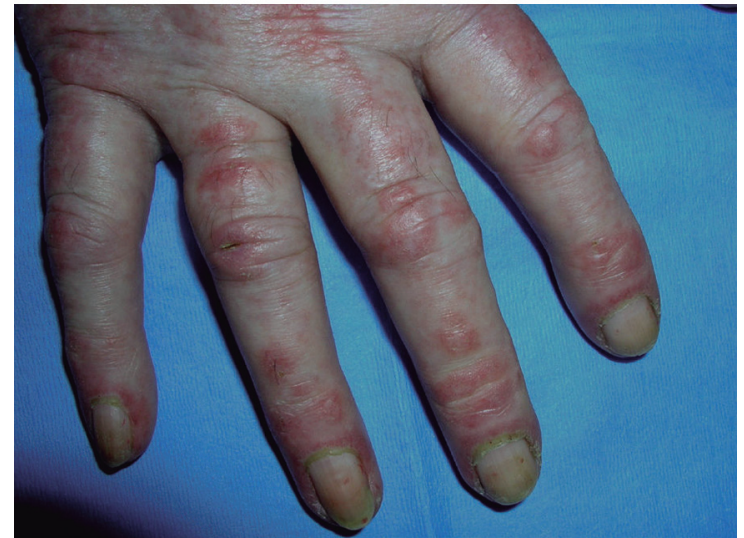

Figura 2: Pápulas de Gottron en articulaciones interfalángicas Fuente: Ciudad-Blanco C. et al. Actas Dermosifiliogr. 2011;102(6)448.55

Algunas de las anteriores manifestaciones son consideradas patognomónicas de la DM. Como las pápulas de Gottron, que son lesiones maculopapulares de coloración violácea que aparecen en la piel que recubre las articulaciones, principalmente interfalángicas y metacarpofalángicas, pero pueden presentarse en las rodillas o en los codos. Estas lesiones pueden confluir y ocasionar placas violáceas descamativas con telangectasias que se conocen como el signo de Gottron ${ }^{15-18}$.

Otra manifestación que se ha considerado patognomónica es la presencia del rash heliotropo, el cual se caracteriza por ser de coloración similar a las pápulas de Gottron, que se ubica simétricamente sobre los párpados y puede extenderse a otras partes del cuerpo, especialmente la superficie facial o torácica. Estos signos semiológicos son muy importantes pues pueden presentarse en un alto porcentaje de pacientes $(60 \text { a } 70 \%)^{15-18}$.

Cuando la miositis cursa sin la presencia del rash se le denomina polimiositis ${ }^{19}$. Los criterios utilizados más frecuentemente para el diagnóstico y la clasificación de las miopatías inflamatorias son aquellos propuestos por Bohan y Peter (Ver Tabla 1 y Tabla 2).

En el cáncer de mama, la DM puede cursar con un cuadro clínico inicial de edema en miembros superiores, rubor facial, disfonía, astenia, mialgias y fiebre, que puede durar incluso meses $7,8,20$. Es frecuente, como síntoma inicial, la debilidad muscular simétrica de inicio insidioso en las extremidades, que puede cursar incluso desde antes de manifestarse propiamente el tumor. El edema es secundario a la debilidad muscular que no permite un adecuado retorno venoso ${ }^{15}$. 
Tabla 1. Criterios diagnósticos para miopatías inflamatorias de Bohan y Peter. La presencia de los primeros cuatro criterios hace el diagnóstico de polimiositis. Si hay cambios característicos en la piel se realiza el diagnóstico de DM.

\begin{tabular}{|l|l|}
\hline \multicolumn{1}{|c|}{ Criterio } & \multicolumn{1}{c|}{ Definición } \\
\hline $\begin{array}{l}\text { Debilidad muscular } \\
\text { proximal. }\end{array}$ & $\begin{array}{l}\text { Debilidad muscular en la } \\
\text { cintura escapular y pélvica. }\end{array}$ \\
\hline $\begin{array}{l}\text { Enzimas sarcoplásmicas } \\
\text { elevadas. }\end{array}$ & $\begin{array}{l}\text { Enzimas sarcoplásmicas } \\
\text { elevadas. }\end{array}$ \\
\hline $\begin{array}{l}\text { Cambios miopáticos en la } \\
\text { Electromiografía. }\end{array}$ & $\begin{array}{l}\text { Potenciales de acción } \\
\text { características de miopatía. }\end{array}$ \\
\hline Biopsia muscular. & $\begin{array}{l}\text { Inflamación crónica } \\
\text { con degeneración y } \\
\text { regeneración de fibras } \\
\text { musculares. }\end{array}$ \\
\hline $\begin{array}{l}\text { Pápulas de Gottron o Rash } \\
\text { heliotropo. }\end{array}$ & $\begin{array}{l}\text { Pápulas planas en los } \\
\text { nudillos de los dedos. Rash } \\
\text { hiperémico alrededor de los } \\
\text { párpados. }\end{array}$ \\
\hline
\end{tabular}

Fuente: Bautista W, et al. Rev Fac Med.2010; 18(2):241-247

Tabla 2. Clasificación de las miositis inflamatorias de Bohan y Peter.

\begin{tabular}{|l|l|}
\hline Grupo 1 & $\begin{array}{l}\text { Polimiositis Idiopática } \\
\text { Primaria. }\end{array}$ \\
\hline Grupo 2 & $\begin{array}{l}\text { Dermatomiositis Idiopática } \\
\text { Primaria. }\end{array}$ \\
\hline Grupo 3 & $\begin{array}{l}\text { Dermatomiositis (o } \\
\text { Polimiositis) asociada a } \\
\text { Neoplasia. }\end{array}$ \\
\hline Grupo 4 & $\begin{array}{l}\text { Dermatomiositis (o } \\
\text { Polimiositis) infantil asociada } \\
\text { a Vasculitis. }\end{array}$ \\
\hline \multirow{3}{*}{ Grupo 5 } & $\begin{array}{l}\text { Polimiositis o } \\
\text { Dermatomiositis asociada } \\
\end{array}$ \\
& $\begin{array}{l}\text { con enfermedad del } \\
\text { colágeno (Síndrome de } \\
\text { Superposición). }\end{array}$ \\
\hline
\end{tabular}

Fuente: Bautista W, et al. Rev Fac Med.2010; 18(2):241-247.

En el diagnóstico, los niveles elevados de creatinquinasa pueden orientar la sospecha hacia esta patología. El electromiograma y la biopsia de piel con alteraciones hacen el diagnóstico ${ }^{7,16}$. Una biopsia muscular permite evidenciar necrosis en las células musculares y, además, presencia de infiltrado perifascicular y perivascular?

Aunque estas características se han descrito en diversos estudios de reportes de caso, son pocos los estudios destinados a evaluar la relación y el comportamiento entre la DM y el cáncer de seno.
Uno de los casos es el estudio original retrospectivo realizado por Chun-Nan et al. ${ }^{21}$ realizado durante los años 1990 y 1998, con un análisis de 128 pacientes con cáncer de seno cursando con polimiositis y dermatomiositis, de las cuales 8 tenían esta última. Dicho estudio tenía como objetivo revisar retrospectivamente los datos de las pacientes afectadas para poder comprender mejor el proceso clínico y la relación existente entre ambas enfermedades ${ }^{21}$.

El estudio arrojó como resultado que, en dos casos, la DM precedió el cáncer de seno, en cinco se presentó de forma concomitante, y en una paciente se presentó posterior a la aparición del cáncer de mama. En cuatro pacientes, después de realizarse cirugía para extirpar el tumor mamario, la DM tuvo mejoría clínica hasta solucionarse completamente. Esto concuerda con los resultados reportados en diversos estudios sobre la resolución de la DM posterior a corrección quirúrgica de la neoplasia mamaria' ${ }^{15,21}$.

Es importante resaltar que la dermatomiositis debe ser diferenciada de otras patologías de la piel que suelen presentarse en la paciente con cáncer de mama, como la metástasis a piel, que puede cursar con similares características. Es importante que el clínico tenga esto en cuenta, y solicite exámenes especializados como la biopsia de piel para tener un diagnóstico correcto ${ }^{22}$.

\section{Complicaciones}

La DM puede causar complicaciones en diferentes sistemas del organismo, lo que conlleva a un aumento en la morbimortalidad de los pacientes. El sistema más frecuentemente afectado por estas complicaciones es el sistema respiratorio. Estas pueden agravar el cuadro clínico, ocasionar inestabilidad vital e incluso causar la muerte ${ }^{23}$.

Entre las más frecuentes se encuentra la enfermedad pulmonar intersticial, presente desde un 5 al $65 \%$ de pacientes con DM. Es frecuente que se manifieste con tos y disnea de inicio súbito (síntomas muy inespecíficos). Se puede encontrar al examen físico crépitos basales en velcro. Se orienta el diagnóstico evidenciando un patrón restrictivo en las pruebas de función pulmonar, lo cual es de muy mal pronóstico. El estándar de oro para el diagnóstico es la biopsia pulmonar. Se recomienda tomar imágenes de buena sensibilidad y especificidad para descartar otros procesos como neumonía o neumotórax ${ }^{23}$. 
Otra complicación es la debilidad del diafragma. En ocasiones pasa desapercibida debido a sus manifestaciones inespecíficas lo cual dificulta realizar su diagnóstico. Puede ocasionar un cuadro de disnea, tos y estornudos de bajo tono. Cuando un paciente tiene un diagnóstico previo de DM y curse con esta sintomatología, es prudente realizar una electromiografía de músculo diafragmático, donde se espera encontrar signos de inestabilidad de membrana que indica alteración de la fibra muscular ${ }^{23}$.

También se puede presentar neumotórax como complicación de DM, y se ha reportado en la literatura la asociación con enfisema subcutáneo y neumomediastino. Este cuadro puede presentarse con fiebre, tos, hipoxemia, y dolor en el sitio del enfisema subcutáneo. Estas complicaciones son infrecuentes y pueden llegar a ser mortales. En estos casos, sería conveniente el estudio imagenológico respectivo para hacer el diagnóstico ${ }^{24,25}$.

Otra complicación poco frecuente es la osteonecrosis atraumática. Los sitios donde más frecuentemente se puede encontrar son la cadera, rodilla y hombro. Cuando no es tratada, puede llevar a que el paciente presente osteoartritis con daño importante en la funcionalidad articular. Es ideal que se realice un tamizaje con resonancia magnética nuclear para identificar a tiempo estas alteraciones en los pacientes con cáncer de mama. También se ha estudiado el valor diagnóstico de la resonancia magnética nuclear short tau inversion recovery de cuerpo completo ${ }^{26}$.

Los pacientes también pueden presentar vasculitis necrotizante, que se presenta con una clínica de pápulas eritematosas dolorosas, que tienen un incremento gradual de tamaño y secreción asociada. Cuando se sospeche, se debe realizar biopsia de piel y el diagnóstico se orienta con la presencia alteraciones en la pared de los vasos sanguíneos tales como infiltrado neutrófilo, depósitos de complemento e inmunoglobulina $\mathrm{G}$, edema y necrosis transmural ${ }^{27}$.

Se ha reportado en la literatura una asociación entre la presencia de DM y aumento en la mortalidad en pacientes con cáncer de mama. El estudio de Park $J K$. et $a{ }^{28}$ que comparó una cohorte de pacientes con diferentes tipos de cáncer, asociados o no con diferentes enfermedades reumáticas incluyendo la DM, encontró que, en las pacientes con cáncer de mama, las enfermedades reumáticas incluyendo la
DM se asociaron con un incremento en la mortalidad, específicamente causada por progresión del cáncer. Es por esto que la presencia de DM en una paciente con cáncer de seno debe considerarse como un criterio de mal pronóstico.

También esimportantetener en cuenta un diagnóstico previo de DM al momento de elegir los esquemas de radioterapia para un paciente con cáncer de seno. El estudio de Lin A. et al. ${ }^{29}$ comparó los resultados de la aplicación de radioterapia en pacientes con diferentes enfermedades vasculares del colágeno (incluyendo DM/ PM) frente a un grupo de control, se encontró que la radioterapia en la mama aplicada al grupo con enfermedades vasculares del colágeno tuvo mayor riesgo de toxicidad severa aguda (consistente de descamación de la piel grado 3 según los criterios del Radiation Therapy Oncology Group), definida como aquella presentada en los primeros 90 días posteriores a la aplicación de la radioterapia.

\section{Tratamiento}

Se han descrito como beneficiosas las pautas de manejo no farmacológico, entre las cuales se incluyen la educación del paciente y su familia, terapias de ejercicio desde un momento temprano del diagnóstico basadas principalmente en ejercicios isométricos, isotónicos, concéntricos y excéntricos, de resistencia y ejercicios con carga. Estos han demostrado mejorar la fuerza muscular, disminuir la fatiga, y causar menores contracturas musculares. También es necesario garantizar un adecuado acompañamiento psicológico, con el fin de realizar un tamizaje de depresión asociada a enfermedad sistémica, especialmente en pacientes con cáncer de mama avanzado y de mal pronóstico ${ }^{30,31}$.

El esquema debe ser orientado según las características clínicas y propias del paciente, el tipo de dermatomiositis y la tolerabilidad de los efectos secundarios ${ }^{31}$. El objetivo del tratamiento es eliminar la inflamación, prevenir el daño muscular y permitirle al paciente recuperar la fuerza, con lo cual se mejora su calidad de vida ${ }^{32}$.

Teniendo en cuenta la posible naturaleza autoinmune de la dermatomiositis, las pautas de tratamiento farmacológico se centran en los glucocorticoides. Aunque no se han realizado ensayos clínicos aleatorizados que demuestren su adecuada efectividad específica en el cáncer de 
mama, hay estudios con menor peso epidemiológico con resultados positivos que sustentan su uso $^{31}$. Sin embargo, otros han demostrado que algunos pacientes no responden a la terapia con glucocorticoides o son necesarias dosis elevadas que son altamente tóxicas, razón por cual se han buscado nuevas alternativas como el metotrexato ${ }^{19}$.

Los glucocorticoides administrados durante los primeros dos meses de inicio de la enfermedad presentan mejores resultados que su administración tardía. Aunque no hay consenso exacto sobre las dosis a utilizar en el caso de las dermatomiositis secundarias al cáncer de mama, se ha reportado que dosis de 0,5 a 1,5 mg/kg/día de prednisona pueden dar resultados favorables ${ }^{32}$.

No se ha unificado un tiempo específico de tratamiento, pero se registra en la literatura que puede aplicarse hasta lograr normalización de las enzimas musculares; y de las pruebas de fuerza muscular. Estas últimas pueden normalizarse hasta dos meses posterior al inicio del tratamiento. En las formas agudas y críticas de la enfermedad, donde puede haber un compromiso vital a corto plazo, es recomendable utilizar 1 gramo de metilprednisolona por tres días consecutivos ${ }^{31}$. Así mismo, pacientes que no respondan o toleren el tratamiento con esteroides, el metotrexato ha demostrado ser una buena alternativa terapéutica ${ }^{19}$. Otros medicamentos utilizados para el tratamiento tanto de la polimiositis como la DM son la ciclofosfamida, ciclosporina, micofenolato, bloqueadores del factor de necrosis tumoral e inmunoglobulinas 33,34 .

Debido al grupo etario de nuestros pacientes, se deben administrar los corticoides con cuidado debido a la osteoporosis y la necrosis avascular ósea. Se debe considerar agregar al tratamiento calcio y vitamina D. La hiperglucemia es un problema en diabéticos, y pacientes con síndrome de Cushing ${ }^{31}$.

Para las manifestaciones cutáneas, se recomienda la protección contra el sol con ropas que cubran la mayor parte de la superficie corporal, y protectores solares en crema o aerosol. Se pueden prescribir antihistamínicos sistémicos para apoyar la terapia31. En términos generales, la primera línea para la DM consiste en prednisolona $0,5-1 \mathrm{mg} / \mathrm{kg} /$ día con un máximo de $80 \mathrm{mg} /$ día durante dos a cuatro semanas, que puede asociarse con metotrexato o azatioprina.
Debido a la hepatotoxicidad de la azatioprina, deben realizarse controles de enzimas hepáticas ${ }^{32,34}$. Un estudio comparó los corticosteroides, metotrexato y la azatioprina, y evidenció un aumento en la mortalidad de la población caucásica en la que se utilizaron corticosteroides para el tratamiento. Además, en ambos grupos que recibieron metotrexato o azatioprina la supervivencia fue similar a 5 años, por lo tanto, es difícil establecer ventajas o desventajas entre estos dos medicamentos ${ }^{33}$.

\section{Conclusiones}

La dermatomiositis es una entidad que, aunque infrecuente, se debe tener en cuenta al momento de realizar la anamnesis y el examen semiológico de un paciente con cáncer de mama. Se debe tener especial atención a las manifestaciones musculares como debilidad, y edema de miembros inferiores. Es necesario identificar al examen físico las manifestaciones cutáneas como el rash en heliotropo, edema periorbitario, el signo de Gottron y la presencia de telangectasias.

Para orientar el diagnóstico se requiere la realización de niveles de creatinquinasa, los cuales estarán elevados. También se recomienda la realización del electromiograma y biopsia de piel, las cuales son esenciales para hacer el diagnóstico. Se debe considerar la realización de biopsia muscular, donde se visualizará la necrosis en las células musculares y la presencia de infiltrado perifascicular y perivascular.

Debe realizarse un seguimiento periódico según criterio médico con el fin de buscar alteraciones como la enfermedad pulmonar intersticial, la cual puede comprometer la vida del paciente, y evaluar signos de debilidad muscular del diafragma como dificultad para la respiración. Se recomienda realizar pruebas imagenológicas pulmonares, espirometría y electromiografía de músculos diafragmáticos según sintomatología referida por la paciente.

Se debe realizar acompañamiento psicológico en pacientes con síntomas de ansiedad o depresión. El tratamiento farmacológico consiste en la aplicación de prednisona o metilprednisolona a dosis según necesidad del paciente, asociada o no, a metotrexato o azatioprina. Es necesario realizar el adecuado suplemento de calcio y vitamina D por la alta incidencia de osteoporosis en las mujeres de edad avanzada. 


\section{Conflicto de intereses}

Los autores de este artículo declaran no tener ningún tipo de conflicto de intereses con la información consignada en el mismo.

\section{Agradecimientos}

Nuestros más sinceros agradecimientos a la Universidad Industrial de Santander, por ayudarnos a adquirir los conocimientos básicos necesarios para la realización de esta revisión, al Hospital Universitario de Santander y a sus pacientes, por permitirnos aprender cada día más y aportar la inspiración para crear nuevo conocimiento.

\section{Referencias bibliográficas}

1. Organización mundial de la salud. Cáncer de mama: prevención y control. OMS [Internet]. 2017 [citado 19 de Abril del 2017]. Disponible en: http:/www.who.int/topics/cancer/breastcancer/ es/.

2. Organización panamericana de la salud. Cáncer de mama. OPS [Internet]. 2017 [citado 19 de Abril del 2017]. Disponible en: http://www.paho.org/hq/index.php?option=com_content\&view $=$ category\&id $=3400 \&$ layout $=$ blog\&Itemid $=3639 \&$ lang $=$ es.

3. World health organization. GLOBOCAN 2012: estimated cancer incidence, mortality, and prevalence worldwide in 2012. WHO [Internet]. 2016 [citado 19 de Abril del 2016]. Disponible en: http://globocan.iarc.fr/Pages/fact_sheets_cancer.aspx.

4. Arrospide A, Soto-Gordoa M, Acaiturri T, López-Vivanco G, Abecia L, Mar J. Costo del tratamiento del cáncer de mama por estadío clínico en el País Vasco. Rev. Esp. Salud Pública. 2015;89(1):93-97.

5. Instituto Nacional de Cancerología-ESE. Incidencia, mortalidad y prevalencia de cáncer en Colombia 2007- 2011. INC [Internet]. 2011 [citado 19 de Abril del 2017]. Disponible en: http://www. cancer.gov.co/files/libros/archivos/incidencia1.pdf

6. World health organization (WHO). GLOBOCAN: Cancer Incidence in Five Continents, Vol. XI. [Internet] 2012. [citado el 19 de abril del 2017]. Disponible en: http://ci5.iarc.fr/Default. aspx.

7. Martínez E, Sánchez A, García A, Dueñas R, Lozano A. Dermatomiositis en el diagnóstico del cáncer: carcinoma de mama y de vesícula biliar. Oncología (Barc.).2005;28(4):31-4.

8. Murgić J, Prpić M, Kirac I, Camino-Varela AM, Bolanca A, Kusić Z. Dermatomyositis as paraneoplastic syndrome of peritoneal and ovarian relapse after long-term complete remission in patient with metastatic bilateral breast cancer. Coll Antropol. 2012;36(1):325-9.

9. Song YJ, Wu YF, Fan T. Dermatosis as the Initial Manifestation of Malignant Breast Tumors: Retrospective Analysis of 4 Cases. Breast Care. 2010;5(3):174-6

10. Levine D, Miller S, Al-Dawsari N, Barak O, Gottlieb AB. Paraneoplastic dermatoses associated with gynecologic and breast malignancies. Obstet Gynecol Surv. 2010;65(7):455-61.

11. Miller FW, Cooper RG, Vencovský J, Rider LG, Danko K, Wedderburn LR, et al. Genome-wide association study of dermatomyositis reveals genetic overlap with other autoimmune disorders. Arthritis Rheum. 2013;65(12):3239-47.

12. Zhang CE, Li Y, Wang ZX, Gao JP, Zhang XG, Zuo XB, et al. Variation at HLA-DPB1 is associated with dermatomyositis in Chinese population. J Dermatol. 2016;43(11):1307-13.

13. Blanco MC, Figueroa L, García F, Francis ML, Carrasco A, Correia
MA, et al. Dermatomiositis como síndrome paraneoplásico del carcinoma medular de tiroides esporádico. Rev Venez Endocrinol Metab. 2006;4(3):61-61.

14. Dias LP, Faria AL, Scandiuzzi MM, Inhaia CL, Shida JY, Gebrim LH. A rare case of severe myositis as paraneoplastic syndrome on breast cancer. World J Surg Oncol. 2015;13(1):134-9.

15. Pectasides D, Koumpou M, Gaglia A, Pectasides M, Lambadiari V, Lianos E, et al. Dermatomyositis associated with breast cancer. Anticancer Res. 2006;26(3B):2329-31.

16. Consejo salubridad general de México. Diagnóstico y tratamiento de polimiositis y dermatomiositis. [Internet]. 20** [citado numero de mes de año]. Disponible en: URL:http://www. cenetec.salud.gob.mx/descargas/gpc/CatalogoMaestro/477 GPC Dermatomiositis/GRR_Dermatomiositis_y_Polimiositis.pdf

17. Medsger TA, Oddis CV. Classification and diagnostic criteria for polymyositis and dermatomyositis. JRheumatol.1995;22(4):581-5.

18. Osako T, Ito Y, Morimatsu A, Tada K, Sakurai N, Takahashi S, et al. Flare-up of dermatomyositis along with recurrence of breast cancer. Breast J.2007;13(2):200-202.

19. Noyola JR, Figueroa C, Rodríguez F, Cuéllar R. Tratamiento de la dermatomiositis con metrotexate. Una alternativa terapéutica. Hond. Ped.1995;18(1):3-7.

20. Nishioka M, Igawa K, Yahata Y, Tani M, Katayama I. Simultaneous occurrence of dermatomyositis and systemic sarcoidosis with recurrent breast cancer. J Dermatol.2012;39(5):485-6.

21. Yeh CN, Chen SC, Hwang TL, Chen MF, Liaw CC, Chan HL. Breast carcinoma in patients with dermatomyositis: a retrospective analysis of eight cases. Chang Gung Med J. 2002;25(6):374-80.

22. Seishima M, Shimizu H, Oyama Z. Skin metastasis of breast cancer clinically undistinguished from amyopathic dermatomyositis. Eur J Dermatol. 2001;11(2):131-133.

23. Restrepo M, González LA, Pantoja AE, Ramírez LA, Vásquez GM. Complicaciones pulmonares de las miopatías inflamatorias idiopáticas: a propósito de un caso con miopatía diafragmática. Rev Colomb Reumatol. 2009;16(3):301-309.

24. Bakhshaee M, Jokar MH, Mirfeizi Z, Atabati E, Tarighat S. Subcutaneous emphysema, pneumomediastinum and pneumothorax in a Patient with Dermatomyositis. Iran J Otorhinolaryngol. 2017;29(91):113-116.

25. Allaoui A, Aboudib F, Bouissar W, Echchilali K, Moudatir M, Alaoui FZ, et al. Spontaneous pneumomediastinum: A rare complication of dermatomyositis. Rev Pneumol Clin. 2017;73(5):258-262.

26. Zhen-Guo H, Min-Xing Y, Xiao-Liang C, Ran Y, He C, Bao-Xiang $\mathrm{G}$, et al. Value of whole-body magnetic resonance imaging for screening multifocal osteonecrosis in patients with polymyositis/ dermatomyositis. Br J Radiol. 2017;90(1073):1-34

27. Akbaryan M, Darabi F, Soltani Z. Dermatomyositis leading to necrotizing vasculitis: a perfect response to applied therapy. Int J Biomed Sci. 2016;12(4):125-9.

28. Park JK, Yang JA, Ahn EY, Chang SH, Song YW, Curtis JR, et al. Survival rates of cancer patients with and without rheumatic disease: a retrospective cohort analysis. BMC Cancer. 2016;16(381):1-9.

29. Lin A, Abu-Isa E, Griffith KA, Ben-Josef E. Toxicity of radiotherapy in patients with collagen vascular disease. Cancer. 2008;113(3):648-53.

30. Callen JP. Dermatomyositis and multiple malignancies in a patient treated with methotrexate. J Surg Oncol. 1983;24(2):113-6.

31. Fabiani LR. tratamiento de la dermatomiositis. scientifica. 2008;6(1):1-4.

32. Martiarena A, Aldamiz-Echebarria M, Martínez C, García JJ. Actualización en el tratamiento de la dermatomiositis: a propósito de un caso. Farm Hosp. 2014;38(6):489-491.

33. Schiopu E, Phillips K, MacDonald PM, Crofford LJ, Somers EC. Predictors of survival in a cohort of patients with polymyositis and dermatomyositis: effect of corticosteroids, methotrexate and azathioprine. Arthritis Res Ther. 2012;14(1):1-9.

34. Mebazaa A, Boussen H, Nouira R, Gamoudi A, Rahal K, Kamoun MR, et al. Dermatomyositis and breast cancer: a multicenter Tunisian retrospective study of 13 cases. Tunis Med. 2011;89(1):18-22. 